\title{
Illustrations
}

Page

I. BAY REGION : TODAY AND YESTERDAY Between 44 and 45

The Bay and its Cities

Copyright, Gabriel Moulin

Golden Gate Bridged by World's

Tallest, Longest Span

Courtesy San Francisco Convention and Tourist Bureau

Peninsula Cliffs

Courtesy San Francisco Peninsula, Inc.

Orchards Carpet the Valleys

Courtesy Redwood Empire Assn.

Mount Tamalpais Looms over Marin County

Courtesy Marvelous Marin, Inc.

The Presidio in 1816

Drawing by Louis Choris
Graveyard, Mission Dolores

Northern California Writers' Project

Northernmost Mission at Sonoma (1824)

Courtesy Redwood Empire Assn.

Russian Chapel at Fort Ross (1812)

Northern California Writers' Project

Vallejo's Casa Grande near Petaluma

Courtesy Redwood Empire Assn.

Pedro Font's Map of San Francisco Bay (I777)

Copyright, Regents of University of California

Between 106 and 107

Mural by Diego Rivera, San Francisco Stock Exchange

Courtesy San Francisco $\mathrm{Mu}$ seum of Art

Golden Gate Bridge Under Construction

Courtesy Standard Oil Company

Water and Power from the Sierra: O'Shaughnessy Dam

Courtesy San Francisco Water Dept.

Steamers Drydocked in Oakland Courtesy San Francisco Chamber of Commerce

Giant Towers Carry 165,000 Volts Across Carquinez Strait Courtesy Pacific Gas and Electric Company
Oil for the World at Point Richmond

Courtesy San Francisco Chamber of Commerce

Sugar Refining at Carquinez Strait

Courtesy C. \& H. Sugar Refining Corp.

Stanford Chapel from the Quad, Palo Alto

Courtesy State Chamber of Commerce

Mills College Art Gallery, Oakland

Courtesy Mills College

Lick Observatory of University of California, Mt. Hamilton

Courtesy Californians Inc. 
II. INDUSTRY : ARTS : LEARNING-continued Page Sather Gate, University of California
Northern California Writers' Project

Sun Yat Sen, in St. Mary's Square-Memorial by Beniamino Bufano

Northern California Writers' Project

III. SAN FRANCISCO'S BY-GONE DAYS Between 136 and 137

American Flag Raised at Yerba Buena ( 1846$)$ Courtesy Wells Fargo Bank \& Union Trust Company

Yerba Buena Cove Crowded with Ships ( 1 849) Courtesy Southern Pacific

Business District in 1852 Courtesy San Francisco Examiner

Execution by Second Vigilance Committee (1856) Courtesy Wells Fargo Bank $\&$ Union Trust Company

Panorama From Russian Hill Courtesy George Fanning

Abandoned Ships on Waterfront Prior to I85I Copyright, Martin Behrman

The First Cable Train (1873) Courtesy J. W. Harris

Shipbuilding South of Rincon Point (1865)

Courtesy Southern Pacific

IV. DOWNTOWN

City Hall

Northern California Writers' Project

Exposition Auditorium

Courtesy U. S. Travel Bureau

San Francisco's Jagged Terraces from the Bay

Copyright, Gabriel Moulin

Skyline from a Sky Window

Courtesy Redwood Empire Assn.

Market Street at 5:15

Northern California Writers' Project
Greenwich Street Cable Car Climbing Telegraph Hill (1884) Martin Behrman Collection

Vallejo Street Wharf in Early Sixties

Courtesy Southern Pacific

Cliff House (1866) Courtesy Southern Pacific

Barbary Coast (1914) Courtesy James Hall

Great Fire of 1906: Looking Down Kearny Street Toward Market Courtesy San Francisco Chronicle

Aftermath of the Great Fire Courtesy San Francisco Chronicle

Ruins of Old St. Mary's Church (I906)

Courtesy Old St. Mary's Church

Between 198 and 199

Labor Day Parade up Market Street

Courtesy San Francisco Chronicle

A Five Minute Walk from Business District

Northern California Writers' Project

Four-Fifty Sutter Building and Sir Francis Drake Hotel

Portsmouth Plaza

Courtesy Redwood Empire Assn. 
I L L U S T R A T I O N S xi

IV. DOWNTOWN-continued

Montgomery Block

Northern California Art Project

\section{STREET SCENES}

California Street Still Challenges the Cable Car

Courtesy State Chamber of Commerce

Chinese New Year Celebration

Northern California Writers' Project

Chinese Children at Thanksgiving Playground Party

Courtesy San Francisco Recreation Commission

Grant Avenue

Northern California Writers Project

Fisherman's Wharf

Courtesy San Francisco Convention and Tourist Bureau

\section{THE CITY'S SIGHTS}

Ferry Building and Boats

Courtesy Southern Pacific

Ships at Dock

Northern California Art Project

Highway and Ocean Beach

Courtesy Redwood Empire Assn.

Panama Pacific International Exposition (1915)

Courtesy Redwood Empire Assn.

Sutro Heights

Aquatic Park

Courtesy San Francisco Convention and Tourist $\mathrm{Bu}$ reau

M. H. De Young Memorial Museum, Golden Gate Park

Northern California Writers' Project
Page

Monument to Robert Louis Stevenson, in Portsmouth Plaza Courtesy Californians Inc.

Between 260 and 26I

Fisherman's Wharf

Courtesy Californians Inc.

SS. Peter and Paul Church Northern California Writers' Project

Pacific Union Club, Mark Hopkins and Fairmont Hotels on Nob Hill

Octagonal House on Russian Hill, built in 1854

Northern California Art Project

Pacific Heights

Northern California Writers' Project

Telegraph Hill from the Precipitous Side

Northern California Writers' Project

Between 322 and 323

Mission Dolores

Courtesy Californians Inc.

Carpenters' Gothic

Northern California Art Project

Bay Windows Catch the Sun

Northern California Art Project

The Pride of Antiquarians (Engine Company No. 15-2150 California Street)

Northern California Writers' Project

U. S. S. California in Drydock at Hunter's Point

Courtesy State Board of Harbor Commissioners

Sea Island Sugar Refinery

Courtesy Sea Island Sugar Company 
VII. ACROSS THE BAY

Pacifica, Goddess of Two Expositions-Sculpture by Ralph Stackpole

Courtesy Golden Gate International Exposition

Clipper in Flight over Treasure Island

Clyde H. Sunderland Photo

Fountain of Western Waters, Golden Gate Exposition

Courtesy Golden Gate International Exposition

Evening Star, in the Court of the Moon

Courtesy Golden Gate International Exposition

Oakland Business District from Lake Merritt

Courtesy Oakland Post-En-

Oakland quirer

Courtesy State Chamber of Commerce
Page

Between 416 and 417

University of California in the Seventies Courtesy Oakland Post-Enquirer

Airview, University of California Courtesy State Chamber of Commerce

Oakland Long Wharf, Built in I 871

Courtesy Southern Pacific

Oakland Wharf Terminal of Central Pacific (1878)

Courtesy Southern Pacific

Big Wheeled Newark (1877I92 I )

Courtesy Southern Pacific

Home of Derelicts-Ships and Human Beings

Howard B. Hoffman Photo

Mission San Jose de Guadalupe Northern California Writers' Project

Vineyard in Livemore Valley Courtesy Wine Institute

VIII. NORTH AND SOUTH OF THE GOLDEN GATE

Between 478 and 479

From Skyline Boulevard the Hills Unfold to the Sea

Courtesy San Francisco Peninsula Inc.

Montalvo Foundation of San Francisco Art Association near Saratoga

Courtesy Montalvo Foundation

Skyline Dam and Boulevard at

Crystal Springs Lakes

Courtesy San Francisco Peninsula Inc.

Pigeon Point Lighthouse

Courtesy San Francisco Peninsula Inc.

Raccoon Strait from Sausalito, Marin County

Courtesy Redwood Empire Assn.

Muir Woods National Monument, Marin County

Courtesy Redwood Empire Assn.

In Petrified Forest near Calistoga Courtesy Redwood Empire Assn.

Russian River Playground

Courtesy Redwood Empire Assn.

State Capitol (1853), Benicia

Northern California Writers' Project

Home of Luther Burbank, Santa Rosa

Courtesy Redwood Empire Assn.

Dirigible Hangar, Moffett Field, Sunnyvale

Courtesy San Jose Chamber of Commerce 